\title{
Regional Cortical Anatomy and Clozapine Response in Refractory Schizophrenia
}

William G. Honer, M.D., F.R.C.P.C., Geoffrey N. Smith, Ph.D., Jocelyne S. Lapointe, M.D., F.R.C.P.C., G. William MacEwan, M.D., F.R.C.P.C., Lili Kopala, M.D., F.R.C.P.C., and Siemion Altman, M.D., F.R.C.P.C.

Regional measures of cortical sulcal and ventricular enlargement on computed tomography scan were studied in a clinical sample of patients treated with clozapine. Cortical sulci were significantly enlarged in clozapine nonresponders compared to responders. The Clinical
Global Impressions score at discharge was related to the size of the posterior frontal and lateral temporal sulci, with large sulci predicting a poorer response to clozapine treatment. [Neuropsychopharmacology 13:85-87, 1995]
KEY WORDS: Schizophrenia; Neuroleptics; Computed tomography; Frontal cortex; Temporal cortex

In treating refractory schizophrenia, poor therapeutic response to the antipsychotic drug clozapine was reported related to prefrontal sulcal enlargement but not to lateral ventricular enlargement (Friedman et al. 1991). This finding was of interest in the context of suggestions that the mechanism of action of clozapine might in part depend on serotonin receptor blockade (Meltze 1991). Serotonin receptors were reported decreased in frontal and increased in the temporal cortex in schizophrenia (Joyce et al. 1993; Ohuoha et al. 1993). The present report describes an attempt to replicate in a clinical sample the finding of poor clozapine response related to prefrontal cortical sulcal enlargement, and to study additional cortical and ventricular regions.

From the Departments of Psychiatry (WGH, GNS, GWM, LK, SA) and Radiology (JSL), University of British Columbia, Vancouver, BC, Canada, and the Treatment Refractory Psychosis Program (WGH, GNS, SA), Riverview Hospital, Port Coquitlam, BC, Canada.

Address correspondence to: Dr. W.G. Honer, Department of Psychiatry, Jack Bell Research Centre, 2660 Oak St., Vancouver, BC, Canada, V6H $3 Z 6$.

Received March 8, 1994; revised September 23, 1994; accepted January 6,1995 .

\section{PATIENTS AND METHODS}

The patient sample comprised 39 individuals with schizophrenia and 3 with schizoaffective disorder, similar to the proportion in a previous report (Friedman, et al. 1991). Patients were diagnosed according to DSMIII-R criteria using a best estimate procedure (Smith et al. 1992). None had a history of concurrent substance abuse. All patients had evidence of poor response to adequate doses of neuroleptics for at least six months (level 5 or 6 according to the May et al. 1988 scale of treatment resistance). The patient sample was made up of 31 men and 11 women whose mean $( \pm S D)$ age was $32.6 \pm 7.9$ years, age at illness onset $21.9 \pm 5.1$ years, and duration of illness $11.1 \pm 7.4$ years. The mean number of previous hospitalizations was $5.9 \pm 4.3$.

The admission daily neuroleptic dose was $1165 \pm$ $910 \mathrm{mg}$ chlorpromazine equivalents for the 32 medicated patients. Clozapine treatment was initiated at a dose of $25 \mathrm{mg} /$ day and increased incrementally in open trials. At discharge, when clinical response was considered optimal (mean duration of $17 \pm 11$ [minimum 4] weeks of clozapine treatment), the patient's mean daily dose of clozapine was $410 \pm 180 \mathrm{mg}$.

Patients were rated at admission and discharge using the Clinical Global Impressions (CGI) scale (Guy 1976); the CGI and Improvement scores were also rated at discharge. For purposes of the present study, patients 
were classified as responders if the discharge CGI score was 4 (moderately ill) or better, and the Improvement score was 2 (much improved) or better. Although the other patients may have improved somewhat, for purposes of analysis they were classified as nonresponders.

Computed tomography scans were obtained as part of a comprehensive assessment of each patient, according to standard clinical practice at the referral Department of Radiology. (The scans were angled at $\sim 15^{\circ}$ to the canthomeatal line, with 4 to $5 \mathrm{~mm}$ slices through the posterior fossa and 8 to $10 \mathrm{~mm}$ slices to the vertex.) Each scan was reviewed by a neuroradiologist (JSL), then rated independently by two raters blinded to clinical status (WGH, GNS) using a scale developed to assess clinical and research CT scans (Honer et al. 1994). The scale permits assessment of each cortical and ventricular region of interest (see Figure 1) according to a 1 to 7 rating of severity, using a total of 44 photographs obtained from reference scans. The reference photographs include each ventricular or cortical space, with ratings of $1,3,5$, and 7 indicating increasing enlargement. To minimize floor and ceiling effects, the reference group of scans included normal controls and patients with schizophrenia. The inter-rater reliability (intraclass correlation) of the two raters ranged from 0.77 to 0.91 for the cortical and ventricular regions assessed.

Repeated measures analysis of variance was used to compare the regional cortical and ventricular scores of responders to nonresponders. Also, a total cortical score (sum of five regional scores) and a total ventricular score (sum of four regional scores) were calculated for each patient. These total scores were compared between responders and nonresponders using analysis of variance, with age as a covariate. An alternate approach to assessing the relationship between treatment response and cortical anatomy was to consider the extent to which the regional cortical scores predicted CGI at discharge, assuming a significant contribution of CGI at admission. Multiple regression was used for these analyses, with each of the five cortical sulcal measures and CGI admission used as predictors of CGI at discharge.

\section{RESULTS}

The mean CGI score (admission $=5.2$, SD 0.6) was significantly improved after patients were treated with clozapine (discharge $=3.8$, SD 0.1, paired $t=10.7, p<$ $.0001)$. Fifteen $(36 \%)$ of the patients were classified as responders. Responders and nonresponders did not differ in mean length of treatment with clozapine (responders $=15$ weeks, SD 7; nonresponders $=18$ weeks, SD 14), mean dose at discharge (responders = $430 \mathrm{mg}$, SD 190; nonresponders $=400 \mathrm{mg}$, SD 200),

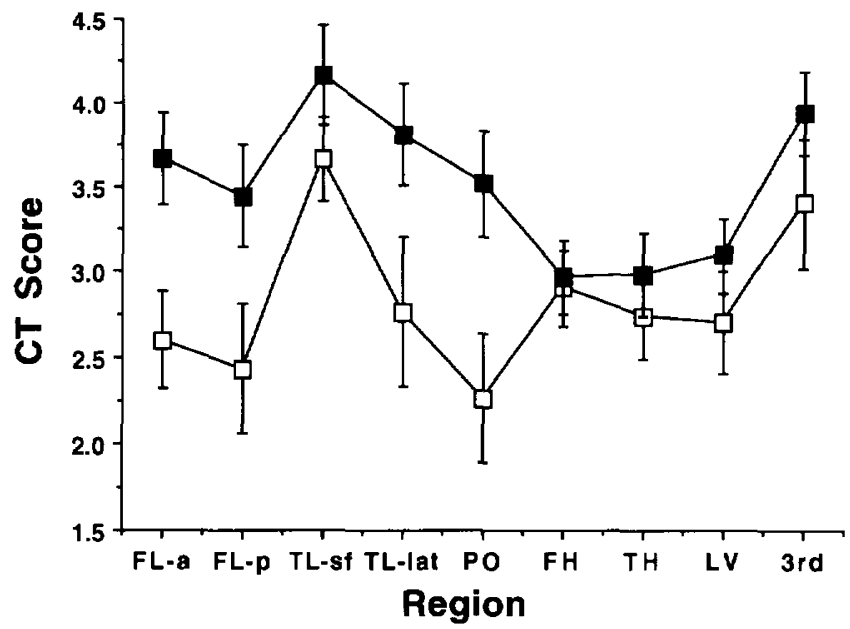

Figure 1. Differences in regional cortical and ventricular enlargement in clozapine responders $(\square)$ and nonresponders (घ). Higher scores indicate enlargement; mean and standard error are indicated. Sulcal regions: FL-a, frontal lobe anterior; FL-p, frontal lobe posterior; TL-sf, temporal lobe Sylvian fissure; TL-lat, temporal lobe lateral; $\mathrm{PO}$, parietal-occipital sulci. Ventricular regions: $\mathrm{FH}$, frontal horn; $\mathrm{TH}$, temporal horn; LV, lateral ventricle; 3rd, third ventricle.

or mean age (responders $=30.7$ years, SD 6.3; nonresponders $=33.7$ years, $\mathrm{SD} 6.3$ )

As seen in Figure 1, the size of cortical sulcal spaces was increased in nonresponders relative to responders $(F=7.08, p=.01)$, while the ventricular spaces appeared comparable in size between the groups $(F=$ $1.43, p=\mathrm{NS}$ ). No significant interaction effects between treatment response and region were observed. Covarying for age, the total cortical score in nonresponders (mean 13.7, SD 5.4) was significantly different from responders (mean 11.7, SD 3.4) $(F=4.32, p<.05)$. Total ventricular scores did not differ between the groups. These results indicate that cortical sulci were globally enlarged in clozapine nonresponders. Multiple regression analysis was computed separately with each of the five cortical sulcal measures and CGI admission as predictors of CGI at discharge. Statistically significant results were observed for the posterior frontal and the lateral temporal sulci. For the posterior frontal sulci, the overall model was significant (multiple $\mathrm{R}=.42, F=$ $4.16, p=.02)$, as was the standardized partial regression coefficient for sulcal rating $(\beta=0.14, t=2.06, p<$ $.05)$. For the lateral temporal sulci, the overall model was significant (multiple $\mathrm{R}=.46, F=5.10, p=.01$ ), as was the standardized partial regression coefficient for sulcal rating ( $\beta=0.16, t=2.45, p=.02)$. In summary, these results indicate that increased size of the posterior frontal and lateral temporal sulci is related to poor response. 


\section{DISCUSSION}

The cardinal result of the present study is that it replicated the findings of Friedman et al. (1991) that prefrontal sulcal enlargement is related to poor response to clozapine. The results also suggest that other cortical regions, in particular the lateral temporal lobe, may influence the capacity to respond to clozapine. Regarding the frontal cortex, receptors for serotonin in this region are reported decreased in schizophrenia and are implicated in mechanisms of clozapine response (Meltzer 1991; Ohuoha et al. 1993). Clozapine reduced frontal cortical metabolism, and the clinical response to clozapine was related to the magnitude of this reduction (Potkin et al. 1993). Animal studies also suggest a role for frontal mechanisms in the response to clozapine. Acute administration of clozapine in the rat leads to induction of the immediate early gene $c$-fos in the frontal cortex, which triggers a molecular cascade possibly related to treatment response (Robertson and Fibiger 1992). Although there is less evidence implicating the lateral temporal cortex in clozapine response, increasing numbers of reports indicate that structural abnormalities in this region correlate with symptom severity in schizophrenia (Barta et al. 1990; Shenton et al. 1992; Nestor et al. 1993). Serotonin receptors are reported increased in the temporal cortex, without changes in reuptake sites, in schizophrenia (Joyce et al. 1993). Further study of frontal and possibly temporal cortical mechanisms related to clozapine response may be important in developing new antipsychotic medications.

\section{ACKNOWLEDGMENTS}

WGH was a Scholar of the Medical Research Council of Canada. This research was supported by Sandoz Canada. The assistance of the nursing and ward staff of the Treatment Refractory Psychosis Unit of Riverview Hospital and the Schizophrenia Research Unit of the Vancouver Hospital and Health Sciences Centre is sincerely appreciated.

\section{REFERENCES}

Barta PE, Pearlson GD, Powers RE, Richards SS, Tune LE (1990): Auditory hallucinations and smaller superior tem- poral gyral volume in schizophrenia. Am J Psychiatry 147:1457-1462

Friedman L, Knutson L, Shurell M, Meltzer HY (1991): Prefrontal sulcal prominence is inversely related to response to clozapine in schizophrenia. Biol Psychiatry 29:865-877

Guy W (1976): ECDEU Assessment Manual for Psychopharmacology, rev. Publication ADM 76-338. Rockville Pike, MD: US Department of Health, Education, and Welfare

Honer WG, Bassett AS, Smith GN, Lapointe JS, Falkai P (1994): Temporal lobe abnormalities in multi-generational families with schizophrenia. Biol Psychiatry 36:737-743

Joyce JN, Shane A, Lexow N, Winokur A, Casanova MF, Kleinman JE (1993): Serotonin uptake sites and serotonin receptors are altered in the limbic system of schizophrenics. Neuropsychopharmacology 8:315-336

May P, Dencker S, Hubbard J, Midha K, Liberman R (1988): A systematic approach to treatment resistance in schizophrenic disorders. In Dencker S, Kulhanek F (eds), Treatment Resistance in Schizophrenia. Braunschweig/ Weisbaden, Vieweg, pp 22-33

Meltzer HY (1991): The mechanism of action of novel antipsychotic drugs. Schizophr Bull 17:263-287

Nestor PG, Shenton ME, McCarley RW, Haimson J, Smith RS, O'Donnell B, Kimble M, Kikinis R, Jolesz FA (1993): Neuropsychological correlates of MRI temporal lobe abnormalities in schizophrenia. Am J Psychiatry 140: 1849-1855

Ohuoha DC, Hyde TM, Kleinman JE (1993): The role of serotonin in schizophrenia: An overview of the nomenclature, distribution and alterations of serotonin receptors in the central nervous system. Psychopharmacology 112:S5-S15

Potkin SG, Buchsbaum MS, Jin Y, Costa J, Richmond G, Telford J, Tang C, Najafi A, Lottenberg S, Gulaskaram B, Bunney WE (1993): Clozapine markedly affects glucose metabolic rate in the striatum and cortex. Schizophr Res 9:207

Robertson GS, Fibiger HC (1992): Neuroleptics increase c-fos expression in the forebrain: Contrasting effects of haloperidol and clozapine. Neuroscience 46:315-328

Shenton ME, Kikinis R, Jolesz FA, Pollak SD, LeMay M, Wible CG, Hokama H, Martin J, Metcalf D, Coleman M, McCarley RW (1992): Abnormalities of the left temporal lobe and thought disorder in schizophrenia. NEngl J Med 327:604-612

Smith GN, MacEwan GW, Ancill RJ, Honer WG, Ehmann TS (1992): Diagnostic confusion in treatment-refractory psychotic patients. J Clin Psychiatry 53:197-200 